\title{
Reconsidering the Architecture of Research in the Public University
}

\author{
Jack C. Schultz
}

Director, Christopher S. Bond Life Sciences Center, University of Missouri

mong the unique strengths of any research university is a community of
faculty members highly skilled and motivated in their scholarly pursuits.
Any research community is highly diverse both in areas of scholarship and approaches to investigation. Many members of the research community also contribute significantly to the education and training of undergraduate and graduate students in classroom and laboratory settings. At the University of Missouri (or, as known locally, MU), the research and teaching missions include more than 1,900 faculty and instructors associated with 286 degree programs.

Maintaining research excellence during difficult times

Maintaining strong research programs during periods of economic hardship is difficult but necessary. Indeed, the National Academies' report "Rising Above the Gathering Storm", issued in 2007, emphasized the need not only for preserving, but revitalizing the nation's investment in science and math education as well as in basic research. That need was reinforced in the University Leadership Council's National Best Practice report, "Competing in the Era of Big Bets" (Education Advisory Board, Washington, DC) which emphasized the importance of multidisciplinary research, especially during perilous economic times.

Both reports, as well as many others, point out that basic research is essential to the nation's ability to maintain productivity and innovation; our economic development depends on basic research. The decline or disappearance of the major industrial laboratories has placed responsibility for conducting basic research in research universities. It is increasingly worrisome that these research universities now have significant competition from other nations even as they experience a relative decline in the core financial support of our public and private universities.

The focus of the ULC's report, Achieving scale in multidisciplinary research, points to an important role for collaborative, interdisciplinary approaches to science in weathering economic storms. Research in many some would say all - of the sciences is increasingly collaborative and interdisciplinary (Wuchty et al. 2007). Solving most modern problems requires more kinds of expertise than single investigators can provide, and the day of 
the lone wolf genius is mostly behind us. One can see this trend in the rapidly increasing fraction of scientific publications with more than one (or two) authors (Wuchty et al. 2007). This trend has even spread to the humanities with the advent of digital publishing (Siemens 2009). Assembling research teams and evaluating their performance has become a science in itself (Guimera et al. 1005, Whitfield 2008).

The best research done in our academic institutions is and will be team-based. This offers a strategy for maintaining and even growing institutional research without greatly expanded resource investment. While no one would minimize the importance of individual team members, the success of interdisciplinary teams depends as much on the mix of members and their interactions as on individuals' traits. Producing emergent properties products and productivity that exceed the sum of individual contributions - is a major justification for forming interdisciplinary research teams. Because the whole can be greater than the sum of its parts, assembling new teams to address different problems can allow institutions to maintain or even excel in research without needing to add major new resources. Engineering careful team building could be an important means of staying ahead in trying times.

\section{Collaborative research as social} network

Teams of collaborating researchers comprise a type of complex evolving network (Barabasi et al. 2002). Working together to solve a research or development problem involves and engages all of the important social interaction principles and skills that any interaction among humans demands. That means that the rules and laws governing networks, most of which apply to any kind of network, are also at work in forming and maintaining research teams (Barabasi et al. 2002). Cooperation is key, and the connections among participants can be encouraged, shaped, and focused on solving any problem. Multi-investigator research collaborations are social networks.

Research networks evolve by preferential attachment, with individuals joining on the basis of forming preferred relationships with other individuals in the network (Barabasi et al. 2002). Such a scale-free network develops as a set of motifs which together give the network its shape, or topology (Milo et al. 2002). Analysis indicates that topological features are critical to network function, that different topologies perform various functions differentially, and that these factors can influence how the network innovates (Obstfeld 2005).

For example, in solving the problem of extinguishing a house fire using water from a nearby lake, the optimal (only) topology for a network of firefighters is a straight line. The bucket brigade is the most efficient way to move water from a single source to a single destination. If a second fire were to break out, it is clear that the bucket brigade would no longer be optimal, and we'd have to rearrange our network of firefighters to accomplish this new, more complex task. Other topologies also have optimal applications. For example, spreading a message via telephone is best 


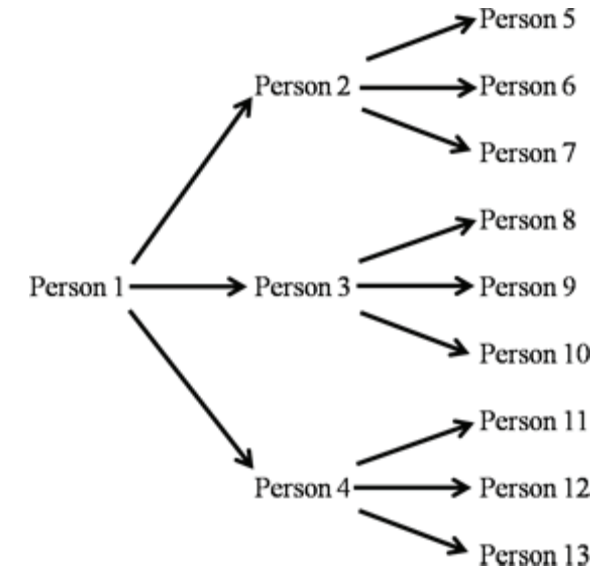

Fig. 1. A phone tree

accomplished with a tree-like network, in which one person calls three, those three each call three more, etc. (Fig. 1). The advantage to the phone tree topology over a bucket brigade in spreading a message is obvious.

Just as certain topologies are ideal for solving certain problems, so does each topology have vulnerabilities (Grubisec et al. 2008). For example, information travels readily between any two adjacent individuals (nodes) in Network A (Fig. 2), but there is no direct connection with non-adjacent nodes. A break between any two nodes severely impacts communication around the network. This problem is resolved in Network B. Network B's greater connectivity produces less impact when a connection is broken.

Breaking links in a network with a topology like Network C produces isolated subnetworks (Fig. 3). Network $\mathrm{C}$ is a hierarchical network, typical of the reporting lines of many organizations, including colleges and universities. Its topology constrains information and other flows among nodes within a level. If communication up and down the network is poor or broken, the end nodes become isolated. This situation is what is referred to as silos in organizations. Lack of connection up and down a topology like $\mathrm{C}$ is almost a default condition in large research universities. Awareness of what is happening at the faculty level does not extend beyond the dean level, if it goes that far. The need for an institution-wide perspective and need to address diverse problems prevents higher administrators from staying abreast of faculty research. As a result, links are certain to be broken and the lower units in the hierarchical network become isolated silos. It becomes commonplace for faculty to be unaware of others on their own campus whose work might benefit theirs, or benefit from theirs. This produces duplicated effort and redundant facilities at significant cost, and most importantly, fails to take advantage of the emergent properties of collaboration.
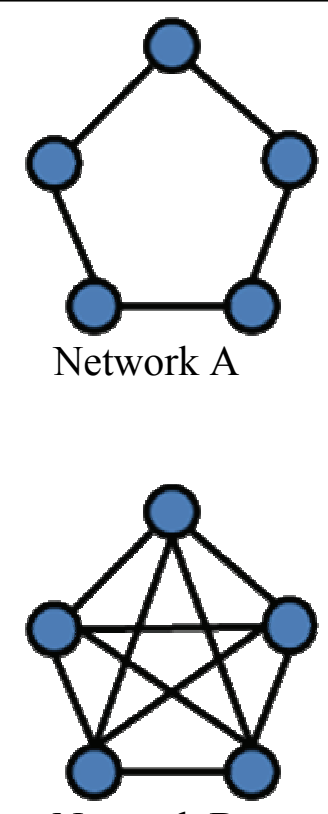

Network B

Fig. 2. Low- and high-connectivity networks. 


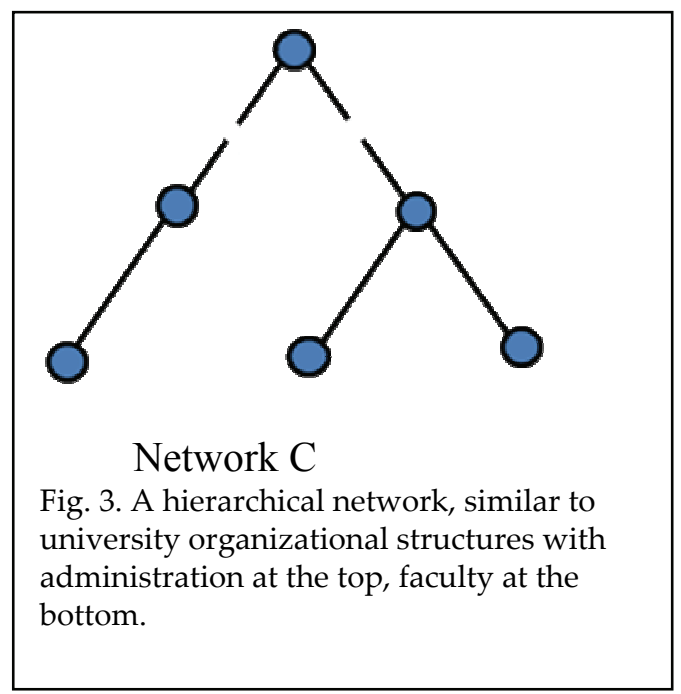

As a result time, effort and money are wasted, something that needs to be avoided as those resources are diminished.

\section{Forming a functional network from} silos

Training has not kept pace with changes in modern life sciences research. Employers and investigators identify at least three shortcomings. First, the culture of research training continues to emphasize individual, independent work. While learning to work independently is important, it is equally important today to learn to function as a team member working with others, often across disciplines. Scientific disciplines and sub-disciplines have their own languages and cultures, and while a student cannot receive deep training all of these, working in a collaborative environment can provide young researchers with important social and communication skills, as well as attitudes, needed for the realities of team science.

A second lack is the ability to use computational tools. This is a longstanding problem in the life sciences, arising in part because quantitative subjects are taught independently of biology from K-12 onward, and because many students interested in biology feel they lack the skills and interest necessary to do well in mathematicallyoriented courses. But the life sciences have always employed statistical and modeling approaches, and today use of bioinformatics has become de rigueur in many areas of biology. There is frequently a cultural and communication barrier separating not only students, but also investigators from biological and computational backgrounds. Indeed, informatics services are springing up at universities to provide computational analyses for life sciences investigators incapable of doing their own.

A third skill set that is almost never addressed in training researchers is the ability to communicate with diverse audiences, including the public. The difficulty researchers have explaining their work to the public is so widely appreciated it has become a stereotype. But in today's economic and social climate, it is more important than ever to explain results and conclusions, as well as their significance to the people who ultimately provide support. Failure to do this well has contributed to a growing view that science in general and research in particular comprise no more than another special interest group.

In 2009 we became aware of the Howard Hughes Medical Institute's Undergraduate Science Education programs, and realized that we might compete for an undergraduate research training grant to address this situation. While preparing any training grant application is a large, complex exercise, 
this one was made more complex by its multidisciplinary nature. The core effort would require the combined expertise of our Bond Life Sciences Center (LSC), the MU Informatics Institute (MUII), and the School of Journalism (SOJ). The LSC exists expressly to foment interdisciplinary collaboration, and it has a history of engaging undergraduates in life sciences research. The MUII is a relatively new organization whose mission includes bringing computational skills to any discipline requiring them, in both education and research. The MU SOJ is nationally-recognized, but in recent years has not invested many resources in science journalism. Finding and engaging interesting SOJ faculty and students (particularly in Strategic Communications, or public relations) would bring excellent communications skills to our research training, and could reinvigorate science journalism on our campus.

To make this coalition work as a coherent program, we also needed expertise in recruiting and managing undergraduates. MU's Life Sciences Undergraduate Research Opportunities Program (LSUROP) exists specifically for this purpose. It manages everything from recruiting to paperwork to housing to locating research laboratories for hundreds of students per year. As do most training grants, the HHMI program requires public outreach, especially to K-12 education. MU's Office of Science Outreach (OSO) is charged with this activity, but its staffing and resources are limited. To extend its reach, we needed the county-by-county organization of the MU Extension system including $4 \mathrm{H}$, and also engaged a statewide high school education organization, Missouri Partnership for Educational Renewal (MPER).

These core activities required participation by individuals and units from colleges and schools that are widely separated on the MU organizational chart. The outreach functions are located in the College of Education and College of Arts and Sciences. The School of Journalism is its own college. The MUII and LSUROP report directly to the Office of Research (which in turn reports to the Chancellor). The LSC is also in the Office of Research, and its faculty are drawn from 12 departments in 6 colleges. The researchers we hoped to engage would be found in any of the dozens of life sciences-related departments on campus. The Bond LSC served as an integrative hub because of its campuswide involvement in life sciences research and outreach.

Preparing the proposal also required cooperation and contributions from a variety of administrative offices. The MU grant-writers' network helped coordinate and collect the information needed for the proposal. Matching funds were provided by the Graduate School, Chancellor's office, and the Vice Provost for Academic Affairs, as well as by core participants. Contact with HHMI was managed by foundation specialists in the Development Office.

Most participants from core units had no prior experience or contact with the other participating organizations. Many had never even thought about what some of the other organizations do. The project PIs pulled together these 


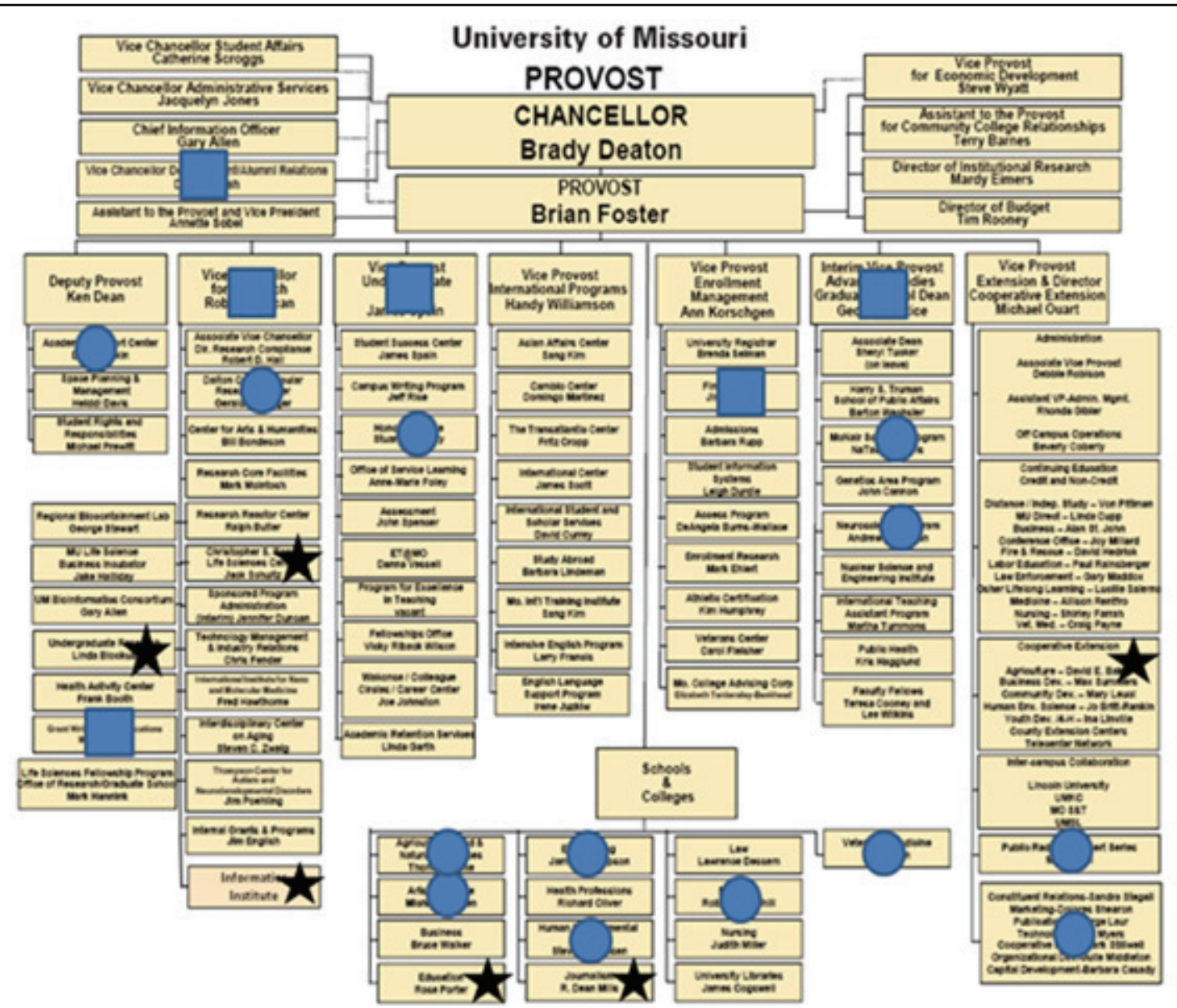

Fig. 4. Organizational chart for MU-Columbia. Stars are core units for HHMI grant proposal; squares are admin units; circles are units from which participants come.

disparate groups via personal interactions, meeting with high school principals, deans, outreach specialists, etc. to gain their participation. In many instances it was not clear who or what resources might be available to achieve a necessary goal. The hierarchical reporting-line organization of the university provided few or no crosslinks among units that needed to come together for this project (Fig. 4). Crosslinking was accomplished for this project by trial-and-error calls, visits, and meetings initiated by the PIs.

In the end, the resulting organization was successful, and the proposal was funded by HHMI. But the network topology required to make the project work is wildly different from the underlying university organizational chart (Fig. 5). Direct links between participating units required for the project to function are widely separated by many links in the university hierarchy. Moreover, many of those hierarchical links are effectively broken, and there may be little or no communication among them. While this may not be surprising - the routine reporting lines cannot be based on every individual project the university may undertake - it also impedes drawing together people and resources from the disparate units needed to make multidisciplinary collaboration work. This is not unique to MU; the hierarchical designs of all university organizations get in the way of meeting major goals, as is also true in the business world. 


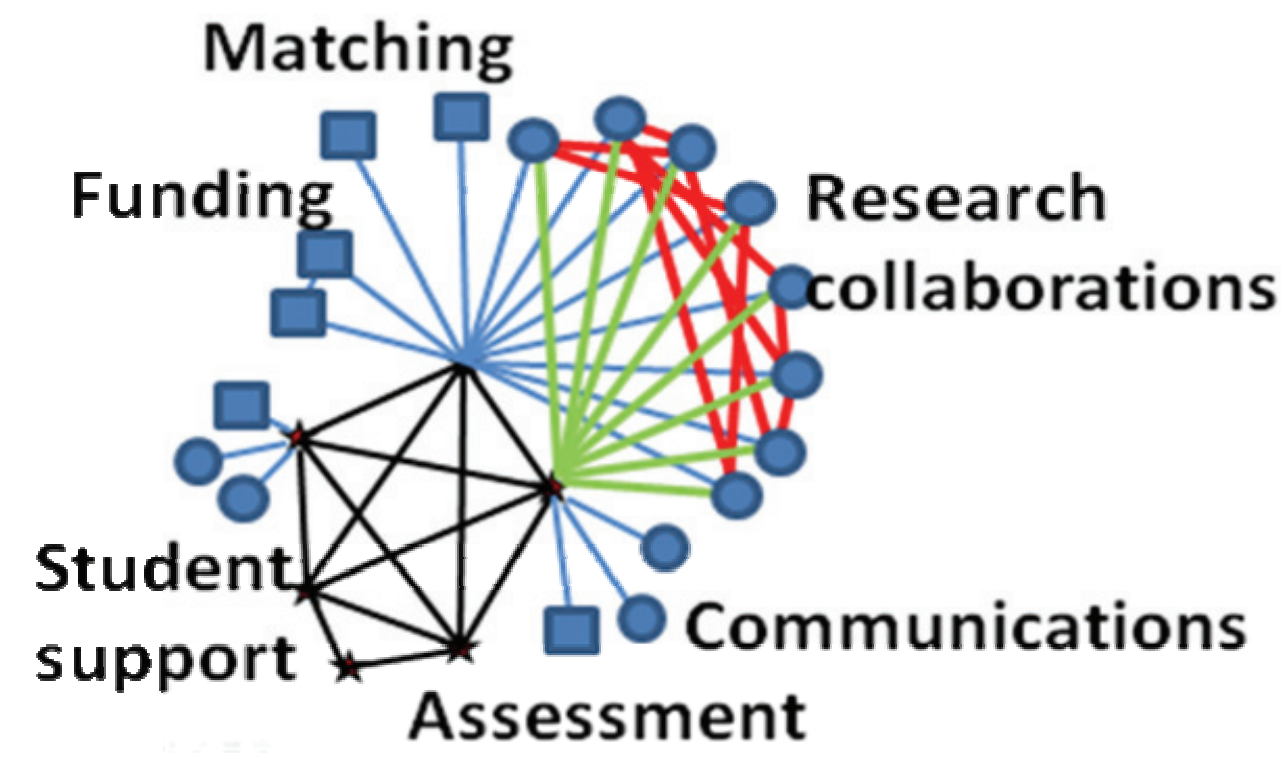

Fig. 5. Actual network assembled for HHMI grant proposal from the MU org chart.

Symbols as in Fig. 4.

\section{Engineering university networks}

The study of the relationship between network topology and function is now well developed. Network analysts have demonstrated common functional relationship between topological details and outcomes for gene regulation networks, transportation networks, social networks, communication networks, and many others (Milo et al. 2002). Similar or identical topological features produce similar or identical outcomes irrespective of the identities of nodes. Stability of networks to perturbation, efficiency of information transfer, and utility for particular tasks can all be associated with particular network topologies. This implies that networks can be designed - engineered - to maximize the likelihood or a particular outcome.

In this discussion of research networks, the assumed connections among nodes (participating researchers) are some form of communication.
Exchanging ideas, data, solutions, etc. is a fundamental aspect of those interactions. Materials, including funds, reagents, instruments, etc. may also be exchanged following network communication lines. While interactions among units on university campuses may be more complex, nothing can be exchanged without communication. So thinking of interactions in university networks primarily as lines of communication (as opposed to responsibility or reporting) seems useful.

But do networks apart from official reporting lines exist on campuses? Many would like to know, and it is currently popular to try to picture research networks using data mined from repositories many campuses develop, such as coauthored publications, collaborative grants, etc. But it is not clear that these products reflect the network interactions that influence outcomes. While coauthored publications probably represent an 
important interaction that shaped an outcome in the past, they do not provide a current view of interactions. Many investigators report that informal interactions that have shaped their work may not culminate in coauthorships. This is certainly true outside the sciences, where sole authorship is the norm, accompanied by lengthy acknowledgements. A lot of research is shaped during informal interaction, even outside of work, which would not be captured by existing incomplete data sets.

Existing university data also are frequently incomplete and out of date. There are many reasons for this, including lack of faculty compliance. Incomplete data not only weaken a network analysis, they may make it impossible. Missing data can eliminate an existing interaction, or even delete an individual from a network graph. This has the effect of changing the rest of the network's apparent topology, which depends on all of the interactions. Missing data do not merely produce a hole in the picture; they can change the entire network picture. Finding out who interacts with whom, and how, is likely to require asking individuals directly, or enticing them to volunteer this information. Unfortunately, existing data sets indicate that voluntary contributions fail to provide the necessary complete information.

So the conundrum is: how can we change a culture of independence to one that recognizes the value of cooperation and information exchange? How can we then shape that culture for maximum impact? A cultural shift like this requires the spread of new attitudes about how we work and what is useful. The first goal needs to be engineering the spread - an epidemic - of new attitudes.

Studies of social networks have shown that the influence of one individual on others follows network lines and has an impact on individuals up to 3 connections away (3 degrees of separation) (Christakis and Fowler 2009). Many attitudes, behaviors, practices and habits follow this rule; one individual's attitude can spread to others over a span of 3 connections (Hill et al. 2010). Once established in those individuals, they begin to have an impact through their connections, so that the original effect spreads through the network, much like a disease. Obesity, smoking and depression are just three of many outcomes that spread in this way (Christakis and Fowler 2009, Rosenquist et al. 2010).

Some people are more connected than are others; they are called "hubs". These hubs are especially important to network function, since they provide more connections along which information can spread. In most networks hubs are relatively uncommon. Because people join networks (unknowingly) by associating with other individuals (as a 'dyad') most interactions have a lower number of immediate connections. Position in a network also confers significance on particular individuals. One person can provide the link between two larger sets, or two topological features in a larger network; these people are connectors.

In a research setting, investigators who are avid collaborators function as hubs, connecting to many others. They may become hubs because of their 
attitude (favoring lots of collaborative activity) or because they are unusually rich in resources (supporting many collaborations) or both. Other individuals may become connectors, perhaps because their interests span interest areas considered to be widely separated (e.g., biology and physics). They may not be in a position to interact with many others, but they do interact with at least one investigator in two otherwise unconnected groups. A crucial trait for an effective connector is the ability to understand two scientific languages or cultures and to 'translate' from one to another.

Hub and connector investigators are highly valuable to a larger research organization, especially where interdisciplinary collaboration is important. They bring different individuals and groups together. And because of their position in the network, they have the opportunity to transmit ideas, approaches and attitudes widely, even creating epidemics. Personality traits are a large component of being a hub or connector, and so should be considered in hiring. Identifying individuals with the attitude and resources that facilitate becoming a hub and placing them into a multidisciplinary environment can create a topology that facilitates collaboration. Individuals may be positioned so that they have the opportunity to form hubs or connections, or interact with influential colleagues. This is, of course, an aspect of what is commonly called mentoring.

Context and physical proximity also are important influences on the spread of influences through a network. A network's setting can influence outcomes significantly. For example body mass index has been shown to be directly related to a neighborhood's density of restaurants (Raja et al. 2010). Placing researchers in facilities of mixed disciplines with an architecture that encourages casual encounters (such as that of MU's Bond Life Sciences Center) is slowly becoming more common. Physical proximity combined with attention to individual attitudes about collaboration, the composition of expertise and interests, and a mix of more- and less-experienced investigators is likely to maximize emergent, novel research outcomes (Whitfield 2008).

The above scenario - multiple interests and attitudes in physical proximity - runs counter to traditional academic organization on most campuses. Researchers are assigned to space on the basis of presumed shared interests and with no regard for whether they will interact. The definition of 'shared interest' is usually defined as a 'discipline' that dates from the formation of the modern university hundreds of years ago, and minimizes crossfertilization with other interests and the evolution of new disciplines. Facilities frequently do not permit, much less encourage, interaction, and the interactions are more like tenants of an apartment building than team mates. Worse, a reluctance to allow subjective criteria in hiring and promotion eliminates the opportunity to exploit and propagate attitudes and approaches that could enhance the quality and productivity of the entire group. But an understanding of network structure and function can help manage research 
architectures, for example by suggesting strategies for dealing with losses (Matisziw et al. 2009).

Achieving a new research architecture

Some way must be found to flatten the typical university organizational structure with respect to interactions among researchers and to build lines of communication across/among units (Cacioppo 2010). This would increase the efficiency of the research enterprise, and the resulting interactions would allow universities to maintain or increase the quality of research programs even as resources become scarce. It is tempting to use electronic means for this; theoretically a search engine could allow anyone to locate and communicate with others having common interests. For those anxious to make connections, this is a worthy goal. But besides the data problems described above, this presupposes a willingness and desire to make this effort. The current culture on our campuses does not favor this effort, because we still train researchers to work alone, and we support that training with promotion and tenure policies that frequently punish collaboration.

Furthermore, academic units (departments, divisions, colleges), treating resource allocation as a zerosum game, perceive inter-unit collaboration as a net loss to them. Coauthorships on papers and grants are thought to dilute the unit's impact and reducing credit accruing to it. This is seen as threatening status on campus and even national rankings. It is therefore not in a unit leader's best interest to encourage interdisciplinary collaboration.

These barriers to a more effective, collaborative research architecture could be overcome by developing new ways of dealing with people, places, and things.

People: Take personality and attitudes into account.

1. Willingness and ability to collaborate or at least work across disciplinary boundaries can be evaluated in new hires. We are often reluctant to use such "soft" criteria in hiring, but when one examines how the behavior of a social network changes, such traits are powerfully influential.

2. Faculty and institutional promotion and tenure committees need to support collaborative research consistently. Institutions must establish policies with respect to how coauthored products are evaluated, and see to it that these are enforced from department to campus-level committees.

Places: Design spaces to put people together so connections are made and concepts spread.

1. Do not assign space on the basis of discipline; that builds silos. Locating researchers on the basis of problems to be solved or other common interests is a promising new idea on university campuses that could become a trend.

2. Design research spaces to encourage informal interactions. Lunch areas or even cafes near research areas keep researchers nearby and encourage conversation. Design meeting spaces of varying 
sizes into research facilities promotes both scheduled and opportunistic meetings.

Things: Provide collaborative tools.

1. Most important is a data base that allows investigators to find each other, or organizers to assemble teams. These need to be kept up to date and edited for consistency.

2. Nothing potentiates the formation of collaboration like sitting at the same table. Actively promoting, by organizing, staffing and funding informal meetings allows one to determine whether new interest clusters are viable or not. While this can be done electronically, face-to-face is far more effective.

3. Foster credit- and resourcesharing among academic units so that a win for one is a win for both. Allow shared credit and doublecounting on grants. Make sure that all units sharing in a success are acknowledged.

These approaches may defy current values and practices on many campuses, and they require a cultural shift. But with the exception of space assignments, accomplishing them is relatively straightforward and uncontroversial. The key is that once in place, network interactions will spread successful interactions, attitudes, and practices without overt top-down forcing. Arrange the landscape and the network will do the rest, including selecting a topology that maximizes research quality, productivity, and innovation, even in times of tight resources.

\section{References cited}

Barabasi, A.L., Jeonga, H., Neda, Z., Ravasza, E., A. Schubert, A. and T. Vicsek. 2002. Evolution of the social network of scientific collaborations. Physica A. 311: 590-614.

Cacioppo, J.T. 2010. To Keep the Engine of Research Humming, Higher Education Must Rethink Its Organization. Chron. Higher Ed. May 23.

Christakis, N.A. and J. H. Fowler. 2009. Connected: The Surprising Power of Our Social Networks and How They Shape Our Lives. Little, Brown \& Co. New York. 352pp.

Grubesic, T.H., Matisziw, T.C., Murray, A.T. and D. Snediker. 2008. Comparative approaches for assessing network vulnerability. Intern. Regional Sci. Rev. 32: 88-112.

Guimera, R., Uzzi, B., Spiro, J., and L. A. Nunes Amaral. 2005. Team Assembly Mechanisms Determine Collaboration Network Structure and Team Performance. Science 308: 697-702.

Hill, A.L., Rand, D.G., Nowak, M.G. and N.A. Christakis. 2010. Emotions as Infectious Diseases in a Large Social Network: The SISa Model. Proc. R Soc. B published online before print, doi:10.1098/rspb.2010.1217

Matisziw, T.C., Murray, A.T. and T. H. Grubesic. 2009. Strategic Network Restoration. Netw. Spat. Econ. 10: 345-361.

Milo, R., Shen-Orr, S., Itzkovitz, S., Kashtan, N., Chklovskii, D., and U. Alon. 2002. Network Motifs: Simple Building Blocks of Complex Networks. Science 298:824-827.

Obstfeld, D. 2005. Social Networks, the Tertius lungens Orientation, and Involvement in Innovation. Admin. Sci. Quart., 50: 100-130.

Raja, S., Yin, L., Roemmich, J., Changxing Ma, C., Esptein, L., Yadav, P. and A. B. Ticoalu. 2010. Food Environment, Built Environment, and Women's BMI: Evidence from Erie County, New York. J. Planning Educ. Res. 29: 444-460.

Rosenquist, J.N., Fowler, J.H., and N.A. Christakis. 2010. Social Network Determinants of Depression. Molec. Psychiatry, forthcoming.

Siemens, L. 2009. "It's a team if you use "reply all" ': An exploration of research teams in digital humanities environments. Lit. Ling. Comput. 24:225-233.

Taylor, M.C. 2009. End the University as We Know It. NY Times Op-Ed, April 27.

Whitfield, J. 2008. Group Theory. Nature 455: 720723.

Wuchty, S., B.F. Jones, and B. Uzzi. 2007. The Increasing Dominance of Teams in Production of Knowledge. Science 316: 1036-1039. 